\title{
Research on the Problems and Countermeasures of Infection Management in XX Hospital
}

\author{
Zejuan $\operatorname{Ren}^{1, \text { a }}$, Xue Sun ${ }^{2, b}$ \\ ${ }^{12}$ Southwest Medical University, College of Humanities and Management, Luzhou, Sichuan, China, \\ 646000 \\ ${ }^{\text {a email, }}{ }^{\mathrm{b}}$ email
}

Keywords: Problems, Countermeasures, Infection Management

\begin{abstract}
As one of an outstanding and global public health problem, hospital infection management gains much attention. Large comprehensive hospitals carry out the infection management work as a focus, and have achieved remarkable results. However infection management work has not be taken into account a lot in primary hospitals, due to various both subjective and objective factors, and its effect is often unsatisfactory too. This paper tries to grasp the basic conditions of the infection management work in XX Hospital and to find out prominent problems, by methods of questionnaires and interviews. Finally, it proposes five pieces of suggestion to these problems as follows: enhancing infection control awareness; improving hospital's infection management organization system; increasing investment to strengthen infection management infrastructure; strengthening management of antimicrobial drug usage; and enhancing training to improve expertise on infection management. It is desirable to provide some references for infection management in primary hospitals.
\end{abstract}

\section{Introduction}

With the rapid development of modern medicine and science and technology, it brings about a wider usage of various types of antibiotics, more various catheters and endoscopes in medical examination and treatment programs frequently; much more chemotherapy and radiotherapy in medical treatments as for a high incidence of malignant trend. These are the most important factors of hospital infection prevalence and infection management becomes one of the tough problems in hospital management. As a principal line of defense for health of grassroots people, the effect on infection management is closely associated with the patient as well as medical personnel. Moreover, it affects the level of medical technology and quality for primary hospitals to a large extent. Therefore, the primary hospitals should attach great importance to the hospital infection management, improve its management level and bring benefit to the local people.

\section{The Existing Problems}

By a comprehensive survey and interview, there comes many problems in XX hospital's infection management, and the most prominent five ones are as follows: 
2.1 The Lack of Infection Control Awareness for Whole Staff. Firstly, Hospital leadership pays inadequate attention to infection management. The carrying out of Hospital infection management and control work requires a lot of investment in human, financial and material resources, but it cannot bring direct economic benefits (for example: the consumption of disinfection and isolation belongs to the hospital's operating costs and expenses spending that cannot expenditure costs from patients). In addition, hospital's inputs on infection management is far less than needed, owning to cost pressures and principalship of economic benefits. As a result, the relevant personnel, capital and management measures were unable to landing. It is believed that the limited power for Hospital infection management deployment comes from correlated examination of superior departments.

Secondly, Hospital infection management's deployment requires close cooperation and support from all levels of the whole staff and different departments too. However, because of disconsider by the leadership, it is no doubt that the staff gets an idea of "infection management is dispensable" naturally. Therefore, the personnel did not strictly execute relevant rules and regulations on infection management. They just "going through the motions" .Actually, this is what permeates

In the survey it is found that some medical personnel has a poor hand hygiene, simplifies hand-washing step just afraid of troublesome, and does not strictly comply with the "six-step hand-washing method". Some nurses, especially the younger ones, whose sterile awareness is not strong, tend to no use of protective equipment or lack of standardization. A few nursing workers have non-standard operation in medical waste treatment too.

2.2 The Unsound Organization of Hospital Infection Management. The hospital infection management committee has not been established yet; there is only one hospital infection management office, with a staff member, who has no professional background related to infection management and a work experience in infection just less than one year. There are no infection management teams in clinical departments with none full-time staff. This unsound organization of hospital infection management causes infection control working carrying out irregularly and no guarantee for its effectiveness.

2.3 Backward in Hospital Infection Management Facilities. Results from site survey show that: The hospital does not have a dedicated cleaning and disinfection room, disinfection and sterilization facilities there are not complete but outdated; equipment in laundry room as types of pool and boiling device are outmoded, existing a risk of infection; monitoring equipment don't match; there is no monitoring on using disinfectant, the concentration of the sterilization and dye bacterium situation, and no B - D tests in prevacuum autoclave as well; the hospital does not set the convenient quick hand disinfection, paper towels access, and express disinfectant for hand-washing is also not fully used, all due to the limited budget. 


\subsection{The High Usage Rate of Antibiotics and Low Criterion of Medication Normative.}

Doctors are suspected of abusing of antibiotics, such as drug combination is used for preventive use and a frequent replacement of a variety of antibiotics when an antibiotic effect is not obvious; three-drug therapy and over triple combinations are based on no more etiology; treatment is too short or too long and so on. In particular, some "senior doctor" tends to be experience-directed, and has a apparent randomness in medication, with no susceptibility test. There are some doctors, considering for their own economic interests, use the more advanced, expensive, imported antibiotics at the very beginning.

Management on usage of antibiotics in this hospital is pretty loose: there are no strict regulations, so that the cost for overuse of antibiotics is quite low referring to physicians here, which leads to a further increased usage of antibiotics.

\subsection{A Lack of Expertise and Training in Infection Management for the Staff.}

The full-time staff in infection management office is lack of expertise, with no professional background, and does not master the latest technical standard for disinfection; meanwhile he is not familiar with. policies and regulations on the national infection management. As for the other medical personnel whose expertise related are scarcer do not grasp the "Hospital Infection Management Approach", "Technical Standard For Disinfection", "Medical Waste Management Method" and other norms very well. The factors above lead to a weak foundation for effective infection management.

On the other hand, staff training on hospital infection management is neglected, going with an insufficient budget. Although there are a few training programs, with incomprehensive content, the staff are not forced to participate, following the principle of voluntariness. Therefore, their enthusiasms are not to be high and the training effect is pretty poor, with a low participation rate.

\section{The Countermeasures}

3.1 Enhance the Infection Control Awareness of Whole Staff. Most important of all, the top hospital leadership should establish a strong sense of infection control consciousness. The leadership must have a better understanding of huge economic losses possibly bringing by hospital infection, especially immeasurable indirect economic loss. It is believed that the hospital infection management level does not decide physical and mental health conditions of patients as well as their medical expenses directly, but also has a significant impact on health and efficiency of medical staff, which in turn relates to outcomes and quality of medical care. In other words, the hospital infection management level can directly reflect the level of hospital management, medical quality, medical technology and ethics. ${ }^{[1]}$ Therefore, it must attach great importance to hospital infection management and ensure its system in normal running. 
Secondly, under the influence of leadership personally, all the medical staff fulfill their responsibilities actively in the hospital infection management, self-monitoring, self-controlling and self-management, to make infection control awareness carrying out on every task.

3.2 Improve Hospital Infection Management Organization System. Considering mature infection management experience from class III comprehensive hospitals, XX Hospital can build up a three level system of the Hospital Infection Management Committee, the Infection Control Branch (office) and clinical monitoring groups. ${ }^{[2]}$ In this system, the Hospital Infection Management Committee belongs to a decision-making body, whose main responsibility is developing infection management strategy, directing and supervising the conduct of infection management work. Infection Control Branch (office) is responsible for the implementation of decisions from Infection Management Committee and developing regulations and related rewards and penalties about hospital infection management. Clinical monitoring teams of each department taking in charge of directors or nurse-managers are expected to be combined with specific circumstances of their own departments to carry out infection control work. All departments are supposed to be in close coordination and mutual support to promote infection management fully.

3.3 Increase Investment to Strengthen Infection Management Infrastructure. The hospital can make improvements on infection management infrastructure from the following aspects: increase budget on infection management to increase the human, material and financial investment; establish special cleaning and disinfection chamber and update sterilization facilities; be equipped with the necessary monitoring equipment to ensure the quality of disinfection and sterilization and the prevention of hospital infection; set the convenient quick hand disinfection, paper towels access and so on in scope of the whole hospital and express disinfectant for hand-washing used fully. ${ }^{[3]}$

3.4 Reinforce the Management on the Usage of Antimicrobial Agents. Strict rules on abuse of antibiotics act should be formulated regulate the use of antibiotics, on purpose of increasing the cost of doctors' overuse of antibiotics severely.

Keep a close eye on microbial inspection rate of clinical departments, and make selection for antibiotic mainly based on etiology and susceptibility test results. Reduce the case of the physician medication to be experience-directed as well as the occurrence of resistant strains. ${ }^{[3]}$ Monitor the use of advanced and expensive antibiotics, imposing a more severe punishment on departments and doctors with low microbial inspection rate.

\subsection{Enhance Staff Training to Improve Expertise Level of Infection Management.} Leadership should attach great importance to the management of infection, equipping with infection management professionals, and support professionals to participate in provincial or national academic conferences and training annually in order to master the most cutting-edge information and technology on infection. 
Increase training budget to provide training courses for medical staff, combining the training effect with the performance evaluation monthly or annually in order to enhance their enthusiasm and motivation for participating. The Infection Management Branch (office) should be carefully design training contents and make training plans for medical staff in bathes on infection control knowledge with a purpose of enhancing full awareness to infection control, proficiently mastering laws and regulations of the "Hospital Infection Management Approach", "Technical Standard For disinfection", "Medical Waste Management Method" and get a better understand on the most cutting-edge information. ${ }^{[4]}$

\section{References}

[1] Chen Qiong. The present situation and countermeasures on infusion room's infection management of the primary hospital under the new situation [J]. China Practical Medicine, Vol. 11 (2012) No 33, p.262

[2] Ye Xiangjin. Problems and countermeasures on infection management of hospitals in mountain area [J]. Chinese Journal of Hospital Infection, Vol. 17 (2007) No 3, p.310-311

[3] Yuan Younan. The present situation and monitoring countermeasures on infection management of the primary hospital [J].Chinese Journal of Hospital Infection, Vol. 17 (2007) No 33, p.440-441

[4] Sun Xue. The investigation and analysis of the hospital infection management in hospital ward in XX hospital [J].Chongqing Medical, Vol. 7 (2015) No19, p.2694-2695 